Revue internationale P.M.E.

Économie et gestion de la petite et moyenne entreprise

Revue

internationale

PME

\title{
Pour une approche contingente de la spécificité de la PME
}

\section{Olivier Torrès}

Volume 10, numéro 2, 1997

URI : https://id.erudit.org/iderudit/1009022ar

DOI : https://doi.org/10.7202/1009022ar

Aller au sommaire du numéro

Éditeur(s)

Presses de l’Université du Québec

ISSN

0776-5436 (imprimé)

1918-9699 (numérique)

Découvrir la revue

Citer cet article

Torrès, O. (1997). Pour une approche contingente de la spécificité de la PME. Revue internationale P.M.E., 10(2), 9-43. https://doi.org/10.7202/1009022ar

\section{Résumé de l'article}

La plupart des travaux concernant la PME reposent sur ridée que celle-ci est spécifique (rôle prépondérant du dirigeant, etc.). Partant de là, de multiples travaux débouchent sur des typologies ad hoc. Toutefois, l'affirmation répétée de cette thèse pourrait laisser entendre que toutes les PME sont spécifiques. La spécificité est alors érigée en principe universel. Or, il ne faut pas oublier que l'entreprise de faible taille peut relever théoriquement d'un mode de fonctionnement qui ne correspond pas au modèle traditionnel de la PME. Autrement dit, une PME peut ne plus être spécifique. Aussi, l'auteur préconise l'adoption d'une approche contingente de la spécificité de la PME qui permettrait de cerner le cadre de validité de cette thèse et de définir les frontières d'une discipline à part entière. 


\title{
Pour une approche contingente de la spécificité de la PME
}

\author{
Olivier TORRÈS \\ Université Paul-Valéry de Montpellier III
}

MOTS CLÉS

\section{Concept de PME - Spécificité de la PME - Universalisme Diversité des PME - Contingence - Dénaturation de la PME Réfutabilité - Évolution de la recherche en PME}

\begin{abstract}
RÉSUMÉ
La plupart des travaux concernant la PME reposent sur l'idée que celle-ci est spécifique (rôle prépondérant du dirigeant, etc.). Partant de là, de multiples travaux débouchent sur des typologies ad hoc. Toutefois, l'affirmation répétée de cette thèse pourrait laisser entendre que toutes les PME sont spécifiques. La spécificité est alors érigée en principe universel. Or, il ne faut pas oublier que l'entreprise de faible taille peut relever théoriquement d'un mode de fonctionnement qui ne correspond pas au modèle traditionnel de la PME. Autrement dit, une PME peut ne plus être spécifique. Aussi, l'auteur préconise l'adoption d'une approche contingente de la spécificité de la PME qui permettrait de cerner le cadre de validité de cette thèse et de définir les frontières d'une discipline à part entière.
\end{abstract}

\section{L'AUTEUR}

Olivier Torrès détient un doctorat en sciences de gestion et il est maître de conférences à l'Université Paul-Valéry de Montpellier III où il enseigne le management international et la théorie des organisations. II est membre de l'ERFI-Montpellier I au sein duquel il poursuit ses activités de recherche sur le thème des stratégies de globalisation des PME. Adresse : ERFI, Université de Montpellier I, avenue de la Mer, Espace Richter - Bât. E B.P. 9659, 34054 Montpellier Cedex 1, France. 


\begin{abstract}
Most of research works about small and medium enterprise are based on the idea that it is specific (essential role of the owner/manager, etc.). Thus, many works result in ad hoc typologies. However, systematically applying this thesis to research could lead to the idea that all SMEs are specific. Specificity would then become a universal principle. Nevertheless, one should keep in mind that SME may refer in theory to a mode of organisation which does not fit the traditional pattern. In other words, a small enterprise may not be specific. As a consequence, the author suggests a contingent approach of SME specificity, which would give an opportunity to define the limits in which this theory remains fit.
\end{abstract}

\title{
RESUMEN
}

La mayor parte de los trabajos de investigacion relativos a la PyME se basan sobre la idea de que esta empresa es especifica (papel preponderante del dirigente...). A partir de ahi, muchos trabajos desembocan en tipologias ad hoc. Sin embargo, una afirmacion excesiva de esta tesis podria llevar a pensar que todas las PyMEs son especificas. La especificidad se erige, pues, como un principio universal. Pero no hay que olvidar que las empresas de pequeña dimension pueden relacionarse teoricamente a un modo de funcionamento que no corresponde al clasico modelo de la PyME. Es decir que una PyME puede no ser especifica. En este sentido, el presente articulo preconiza la adopcion de una posicion que pone en duda la especificidad de la PyME. Se trata aqui de comprender los limites de validez de dicha tesis y de definir las fronteras de una disciplina considerada como tal.

"C'est notre tendance à rechercher la régularité des occurrences et à prescrire des lois à la nature qui est à l'origine du phénomène psychologique de la pensée dogmatique ou, plus généralement, du comportement dogmatique: nous présumons partout la régularité, et nous nous efforçons de la trouver même là où elle n'existe pas ; [...]»

K. POPPER

\section{Introduction}

À en juger par le nombre de colloques et de thèses de doctorat lui étant consacrées, la recherche en PME est en plein essor. À titre d'illustration, sur le seul thème du développement international des PME, sans prétendre à l'exhaustivité, cinq colloques ont été organisés depuis le début des années 1990 (mai 1992, Montréal ; juin 1993, Aix-en-Provence ; octobre 1993, Carthage ; octobre 1993, Moncton ; juin 1994, Strasbourg). En ce qui concerne les thèses de doctorat soutenues en France entre 1992 et 1993 dans la discipline du management stratégique, à peine $10 \%$ des recherches se fondent sur la grande entreprise comme lieu spécifique d'investigation tandis que $25 \%$ des chercheurs ont porté un intérêt exclusif aux 
PME (Bernard, 1994). La recherche en PME se développe de plus en plus et, par conséquent, se structure et s'organise de mieux en mieux.

En effet, le nombre de revues scientifiques consacrées à la PME et à l'entrepreneuriat ne cesse de croître (Julien, 1994). Si la première revue date de 1952 (Internationales Gewerbearchiv. Zeitschrift fur Klein und Mittelunternehmen), c'est dans les années 1980 que la plupart des revues spécialisées ont été créées (International Journal of Small Business, 1982 ; Journal of Small Business and Entrepreneurship, 1982; Journal of Entrepreneurship and Regional Development, 1987 ; Revue internationale PME, 1988 ; Piccola Empresa, 1988 ; Small Business Economics, 1989...). De plus, toujours selon P.A. Julien, « dans la dernière décennie, ce fut l'explosion avec la multiplication d'équipes de recherche de toutes tailles spécialisées dans le domaine de la PME ». Le milieu de la recherche en PME se structure de plus en plus, notamment par le biais de laboratoires, mais également par celui de certaines associations comme le Conseil international de la petite entreprise (ICSB) qui organise régulièrement des colloques à travers le monde entier. De même, les récents colloques internationaux francophones de la PME témoignent de l'essor et de la vigueur du « réseau PME » dans les pays de langue française.

Qu'est-ce qui amène les chercheurs à s'intéresser exclusivement aux PME ? Schématiquement, il est possible de repérer trois types de justifications concernant la recherche exclusive en PME:

- La justification empirique : la PME comme champ d'analyse

Les PME occupent une place importante dans la plupart des économies. En France, en 1986, les PME de moins de 50 employés représentaient 98,8\% des établissements recensés par l'INSEE et environ la moitié des emplois (Julien et Marchesnay, 1988). La récente création d'un ministère de la PME en France témoigne de l'importance que les élus politiques accordent désormais aux entreprises de petites dimensions. On est loin du gigantisme industriel prôné sous l'ère pompidoulienne. La PME constitue un «enjeu de taille » pour amorcer la lutte contre le chômage comme en témoigne le slogan simpliste de la dernière campagne de Berlusconni en Italie : « trois millions de chômeurs, trois millions d'entreprises ». Ces entreprises à dimension humaine posséderaient toutes les caractéristiques requises pour s'adapter aux situations de crise : souplesse, dynamisme et flexibilité. Le phénomène PME constitue donc un enjeu économique et justifie de ce fait les études qui lui sont consacrées. L'aspect salutaire de la PME présentée souvent comme «modèle d'adaptation à la crise » s'apparente au phénomène du «small is beautiful ». Mais il semble que d'autres raisons peuvent expliquer les recherches sur la PME. 
- La justification méthodologique : la PME comme outil d'analyse

Par sa faible dimension, la PME est souvent présentée comme une unité productive dont les phénomènes sont plus facilement identifiables, plus lisibles (d'Amboise et Maldowney, 1988). Selon Marchesnay (1993), la recherche en PME permet de faire apparaître «concrètement, visiblement aux yeux de l'observateur, ce qui est caché, difficile à saisir et à interpréter dans les organisations de grande dimension». De même Sarnin (1990), à partir d'une enquête visant à cerner l'impact des changements stratégiques sur les politiques de formation en PME, pose la question de savoir quelle est la spécificité de ces éléments par rapport aux grandes entreprises: «Ne sont-ils pas simplement plus facilement appréhendables par l'observateur?» Ainsi, «la pertinence de l'objet PME tient plus dans sa valeur heuristique d'analyse des changements que dans la construction d'une catégorie, d'un concept empirique particulièrement utile » (Sarnin, 1990). L'intérêt du concept PME est d'abord méthodologique dans la mesure où certaines pratiques stratégiques sont plus faciles à percevoir que dans les très grandes entreprises où tout est plus dilué.

- La justification théorique : la PME comme objet d'analyse

Au cours des années 1980, les PME ont acquis un véritable statut en tant qu'objet de recherche scientifique. Quels sent alors les fondements théoriques qui justifient cette évolution?

Sur le strict plan économique, Julien (1993) a recensé plusieurs théories expliquant la présence des petites entreprises. Le rôle de l'entrepreneuriat, la théorie des interstices, les critiques à l'égard des économies d'échelle ou de champ, les besoins de flexibilité et les mutations de nos systèmes productifs sont autant de justifications théoriques qui plaident en faveur d'un renouveau de la théorie économique basée sur l'instabilité plutôt que sur la recherche d'équilibre.

Mais qu'en est-il en sciences de gestion ? Sur quels fondements théoriques les sciences de gestion se basent-elles pour considérer la PME comme un objet ou un champ de recherche ? Ce type de justification paraît être de loin le plus judicieux pour asseoir la légitimité de ce courant de recherche en mal d'identité et de reconnaissance. Afin de répondre à cette multitude d'interrogations théoriques et épistémologiques, nous montrerops, dans une perspective historique, les différentes étapes franchies par le courant de recherche en PME. 


\section{Les prémices (1965-1975): les fondements du découpage selon la taille}

Aussi surprenant que cela puisse paraître, les premiers jalons de la recherche en PME sont à mettre au crédit de chercheurs qui ne travaillent par sur la PME. En effet, nous avons déjà insisté sur l'importance du facteur taille comme critère de découpage. Mais avant de considérer les PME comme des organisations particulières, il a bien fallu montrer que la taille n'était pas un facteur neutre sur le plan organisationnel. Ainsi, selon Brooksbank (1991), avant de définir le concept de «petite entreprise», il convient de répondre à deux questions préalables : qu'est-ce que la taille et comment mesure-t-on la taille ? Où se situe la frontière critique entre les grandes et les petites entreprises?

\subsection{Identification de l'effet-taille}

Parmi les travaux qui ont mis en évidence l'influence de la taille sur l'organisation dans les années 1960, l'école d'Aston (Pugh et al., 1968 ; 1969) est très souvent citée comme pionnière, notamment dans l'amorce d'analyses comparatives visant à découvrir les problèmes communs et spécifiques d'organisations de tous types. Les principaux résultats de l'école d'Aston confortent l'idée selon laquelle « la taille de l'organisation constitue un facteur prédictif majeur de sa structuration » (Desreumaux, 1992). Pour ce qui est de la relation entre la taille et la structure, de nombreux travaux empiriques confirment les résultats du groupe d'Aston (Blau et Schoenherr, 1971 ; Child et Mansfield, 1972, etc.). Selon Blau (1970), la taille est l'un des principaux facteurs de contingence, de contexte. Enfin, selon Mintzberg (1982), la taille est certainement le facteur de contingence le plus unanimement reconnu quant à ses effets sur la structure d'une organisation : «Plus une organisation est de grande taille, plus sa structure est élaborée: plus les tâches y sont spécialisées, plus ses unités sont différenciées, et plus sa composante administrative est développée. Les preuves sont ici écrasantes. » À partir d'une approche nécessairement comparative, ces différents auteurs montrent que les caractéristiques organisationnelles des entreprises sont significativement différentes selon la taille.

Pourtant, « cette convergence ne doit pas faire illusion, d'une part, parce qu'il existe de nombreuses nuances dans les travaux et des différences dans le détail des mesures et dans la composition des échantillons qui rendent les généralisations dangereuses, d'autre part, parce que d'autres travaux empiriques ne sont pas aussi affirmatifs sur le rôle de la taille en matière de structuration » (Desreumaux, 1992). En effet, le rôle et l'importance du facteur taille ne sont pas appréhendés de la même façon selon les auteurs et les époques. Effectuant une synthèse de la littérature à partir de plus de quatre-vingts études concernant la taille et la structure organisationnelle essentiellement sur la période 
1965 / 1975, Kimberly (1976) distingue deux courants aux approches antagonistes : l'approche « intertypique » et l'approche «intratypique».

Durant les années 1970, un vif débat a opposé les chercheurs sur le degré d'homogénéité des échantillons. Suivant l'approche «intertypique», l'effettaille transcenderait largement les différences entre les organisations. Dans ce cas, l'échantillon peut présenter une forte hétérogénéité ; les effets de la taille existent, indépendamment du type d'organisation étudié. Il y aurait donc une universalité de l'effet-taille. Ainsi, Hall, Haas et Johnson (1967) avancent qu'une théorie générale des organisations doit permettre de déduire des hypothèses qui peuvent être testées sur un échantillon hétérogène d'organisations. C'est la raison pour laquelle ils réunissent dans leurs échantillons des organisations aussi diverses qu'une station de télévision, un syndicat, une école religieuse ou un établissement pénal. Il s'agit moins de mettre en évidence le rôle de la taille que son importance à travers les divers types d'organisation. De même, à partir d'une enquête transculturelle, Hickson et al. (1976, dans Kimberly) considèrent que l'importance de la taille est tout autant relative qu'absolue : «Même si les organisations indiennes sont moins formalisées ou moins autonomes que les organisations américaines, il n'en demeure pas moins que les grandes organisations indiennes sont plus formalisées que les petites organisations indiennes. » Hall (1972) pourtant adepte de l'approche «intertypique » reconnaît lui-même qu'il n'y a pas de «lois » concernant la taille et les caractéristiques organisationnelles. Implicitement, les adeptes de l'approche intertypique considèrent que la taille se situe au premier rang de la hiérarchie de l'ensemble des facteurs de contingence. Cette position sera dénoncée comme « un véritable impérialisme de la taille organisationnelle».

À l'inverse, les partisans de l'approche intratypique se fondent sur des échantillons dont les entreprises appartiennent au même type (hôpital, écoles, entreprises publiques, etc.). Ils avancent l'argument selon lequel une théorie des organisations est construite à partir d'analyses empiriques portant sur un type donné d'organisation, et ce n'est qu'ultérieurement qu'elle est testée et validée par réplications sur d'autres types d'organisation (Blau et Schoenherr, 1971). De même, Child (1972) considère qu'il est préférable de constituer des échantillons homogènes fondés sur le même type d'organisation. L'avantage de cette approche est qu'elle facilite la lisibilité de l'effet-taille qui ne peut être brouillée par les effets d'une différence entre les types d'organisation. Mais se pose alors la question de savoir comment définir un type d'organisation. En effet, comment être sûr que les unités de l'échantillon sont homogènes? Plusieurs auteurs se contentent de considérer que le type d'organisation est une catégorie nominale portant la même appellation. Ainsi, les hôpitaux correspondent à un type d'organisation, et les prisons, les écoles, et les entreprises correspondent à d'autres types d'organisation. Mais ces conjectures sont d'autant 
moins acceptables qu'un fort degré de variété peut exister au sein d'une même catégorie d'organisation. Peut-on mettre sur le même plan une école maternelle, primaire, secondaire et supérieure ? Si la constitution d'un échantillon homogène est incontestablement utile sur le plan théorique, elle semble difficilement réalisable sur le plan empirique.

Aucune des deux approches ne fait l'unanimité, car chacune présente son lot d'avantages et d'inconvénients. Le choix en faveur de l'une ou l'autre découle de la conception du chercheur à l'égard du rôle de la taille. La recherche d'un échantillon homogène peut conduire le chercheur à multiplier excessivement le nombre de critères de sélection pour constituer son échantillon. Ce type d'approche présente le risque de déboucher sur l'impossibilité de comparer deux organisations sous prétexte qu'elles présentent la moindre différence. À l'extrême, chaque organisation devient alors un cas particulier. C'est la dérive casuistique. Aucune généralisation n'est possible. Inversement, dans le cas de l'approche « intertypique », le rôle de la taille semble transcender les différences entre les organisations. La taille est alors considérée comme LA variable explicative souveraine puisqu'elle a plus d'importance que n'importe quelle autre variable. C'est la dérive universelle. «Ceci fait que le rôle de la taille sur la structure des organisations reste sujet de controverses. Dans l'état actuel des recherches, on peut considérer que, si la taille a un effet sur la structure, elle ne vaut pas nécessairement pour toutes les variables structurelles et que d'autres facteurs explicatifs sont vraisemblablement à l'œuvre. » (Desreumaux, 1992) De sorte qu'il serait vain d'ériger la taille comme le facteur suprême en excluant l'influence d'autres facteurs. Cette position rejoint pleinement celle de Kimberly (1976) qui propose d'adopter « une approche plus contingente du rôle de la taille en cherchant sous quelles conditions ce rôle pourrait varier ». Cette proposition se situe aux antipodes du courant de l'effet-taille absolu. Si l'on accepte l'idée que le rôle de la taille varie selon le type d'organisation, alors il importe de définir ces différents contextes.

En définitive, au risque d'être caricatural, nous pouvons opposer deux courants de recherche :

- celui qui considère la taille comme «la » variable prédictive par excellence. Au-delà du rôle primordial de la taille, ce courant accorde à ce facteur une importance absolue : l'effet-taille est universel.

- celui qui relativise non seulement l'importance, mais aussi le rôle de la taille. Ces auteurs prônent un examen critique et nuancé dans le but de relativiser la portée universelle de l'effet-taille : l'effet-taille est contingent.

Supposons, afin de progresser dans notre analyse, que le problème de la mesure de la taille soit parfaitement résolu, tant en ce qui concerne le choix du critère que de sa pertinence à l'égard de certaines évolutions actuelles; il 
reste à définir à quel seuil le chercheur doit opérer le découpage entre les entreprises pour distinguer les entreprises selon leur taille. Conformément au cadre posé par Brooksbank (1991), la recherche en PME repose implicitement sur l'idée qu'il existe une frontière entre le monde des PME et celui des grandes entreprises. Où se situe cette frontière ? Quel est le seuil au-delà ou en deçà duquel les configurations organisationnelles peuvent être considérées comme spécifiques? La difficulté est de trouver où se situe exactement la «frontière critique » (Sarnin, 1990), «the dividing line » (Brooksbank, 1991) afin d'opérer le découpage. Ce seuil critique existe-t-il seulement ? Répondre à ces questions suppose qu'il faille considérer la taille non plus comme un facteur dont on mesure l'influence à partir d'analyses statiques comparatives, mais comme une variable appréhendée à partir de modèles dynamiques. Toute une série de recherche vont alors se consacrer à l'étude de la croissance de l'entreprise et proposer des modélisations.

\subsection{L'identification de la frontière critique ${ }^{1}$}

Durant le début des années 1970, tout un courant de recherche va se développer autour d'un objet de recherche commun : la croissance de l'entreprise. L'idée centrale de l'ensemble de ces travaux consiste en la mise en évidence de phénomènes de rupture de l'organisation au fur et à mesure de son développement. Plusieurs auteurs considèrent que les effets de la taille s'exercent comme un processus «marqué par des changements abrupts et discontinus » des structures des organisations et des conditions dans lesquelles elles fonctionnent (Starbuck [1965] dans Mintzberg, 1982, p. 223). Selon Mintzberg, « de nombreux éléments nous indiquent qu'à mesure que les organisations grandissent, elles passent par des périodes de transition structurelle, qui sont des changements de nature plutôt que des changements de degré ». Au fur et à mesure de la croissance de leur taille, les entreprises se transforment, se métamorphosent. On passe de l'évolution de l'entreprise (changement de degré) à la révolution (changement de nature) [Greiner, 1972]. En résumé, la croissance de l'entreprise n'est pas un phénomène continu et rectiligne, mais au contraire discontinu et ponctué par des crises, des métamorphoses. Le changement de degré de la taille s'accompagne d'un changement de nature de l'organisation. Ces modèles de métamorphoses signalent les sauts qualitatifs de l'entreprise au fur et à mesure de son développement. Les changements quantitatifs de la taille s'accompagnent à un moment donné (la situation de crise) d'un changement dans la nature de l'organisation. Si bien que l'on peut considérer que chaque forme d'organisation revêt des spécificités, des caractéristiques propres.

1. Cette partie est grandement inspirée par la synthèse effectuée par A. Godener (1994) concernant les modèles de métamorphoses (voir la bibliographie). 
Toutefois, les transitions entre chaque stade sont plus supposées et théoriques que réellement démontrées sur le plan empirique, tout simplement parce que les périodes de transition sont difficilement mesurables. Elles ne sont que le résultat d'interprétations théoriques. À chaque période correspond un mode de fonctionnement spécifique qui va engendrer une crise spécifique. Mais ces spécificités relèvent du domaine de l'interprétation d'un phénomène qualitatif. L'intérêt est moins d'identifier précisément des seuils critiques que de mettre en garde les dirigeants d'entreprise contre d'éventuelles crises que l'entreprise devra surmonter lors de sa croissance. C'est la raison pour laquelle la plupart des modèles de croissance sont davantage conceptuels que testés empiriquement (Godener, 1994). En définitive, ces modèles apparaissent trop déterministes. «Le vrai problème de l'analyse de l'organisation-PME dans l'optique dynamique est plus de mettre en évidence les seuils critiques que traversent les petites et moyennes entreprises au cours de leur croissance et d'en rechercher les causes que de chercher à déterminer la succession virtuelle des étapes de croissance. » (Gervais, 1978)

Aussi, dans la période des années 1980, plusieurs auteurs vont tenter de valider empiriquement ces modèles de croissance pour mettre en évidence les seuils critiques. Comme on pouvait s'y attendre, les résultats obtenus sont extrêmement flous. Aucun seuil critique n'est identifié précisément. «Brac de la Perrière identifie comme deuxième stade de développement l'étape où l'entreprise comprend entre 50 et 200 personnes, alors que pour Steinmetz, cette même étape correspond à l'entreprise de 30 à $250-300$ personnes ; quant aux phases suivantes, là où Basire voit trois périodes distinctes pour passer d'un effectif de 200 personnes à celui de 1000 , Brac de la Perrière et Steinmetz n'en voient qu'une. » (Godener, 1994) Il n'y a pas de seuils qui fassent l'unanimité, ne serait-ce que parce que les différents organes d'une entreprise évoluent certainement à des rythmes différents et selon des modalités diverses (Godener, 1994). Cette forte hétérogénéité conforte l'idée selon laquelle il y a plus de contingence que de déterminisme dans le processus de croissance des entreprises. Selon Kazanjian et Drazin (1990), la structure mise en place et le mode de fonctionnement adoptés par l'entreprise ne sont que des adaptations à une situation particulière. Aucun modèle ne semble « universellement » admis ni pour autant réfuté. Les différents auteurs concluent à des stades et à des transitions différentes. Le comportement de l'entreprise face à la croissance diffère d'un auteur à l'autre. Si l'existence d'un cycle est largement admise, c'est son degré de généralité qui est plus contesté. Même si Greiner (1972) propose un modèle type de croissance de l'entreprise, il est conscient de ses propres limites. Pour ce dernier, le chemin d'expansion d'une entreprise n'est pas indépendant du type de secteur et de la culture. C'est la raison pour laquelle nous sommes d'avis que Greiner, malgré l'aspect déterministe de son modèle de croissance, ne peut être entièrement considéré comme un partisan de l'approche universelle. 
Après la phase de recherche d'un «modèle universel » de croissance, les recherches actuelles, plus critiques, s'orientent donc vers la prise en compte de l'influence du contexte sur le chemin d'expansion de l'entreprise (Godener, 1994). Il n'y aurait donc pas un modèle de croissance universel, mais plusieurs modèles de croissance adaptés à des situations particulières. Les propos de Godener (1994) résument parfaitement l'évolution de la recherche concernant ces travaux :

[...] la limite la plus fondamentale de ces modèles provient d'une étude de Birley et Westhead (1990) qui démontre que chaque entreprise a son propre chemin de croissance ; cette proposition confirme empiriquement ce que certains suggéraient (Salter, 1970; Gervais, 1978; Kazanjian, 1984) : le modèle des stades de croissance est trop général pour être opérationnel : une entreprise peut, par exemple, avoir simultanément les caractéristiques d'une entreprise de la phase 1 pour l'aspect structure, et les caractéristiques d'une entreprise de la phase 2 pour ce qui concerne la fonction production. Cela ouvre le champ à de nouvelles recherches dont l'objectif ne serait plus de chercher «le » modèle universel, mais plutôt de cerner l'évolution d'entreprises dans une situation particulière donnée.

Ainsi, on retrouve pour l'ensemble de ces travaux le même clivage en matière d'approche qui distingue les travaux sur l'effet-taille. À la dérive dogmatique du courant qui cherche à déterminer LE modèle de croissance universel de l'entreprise s'oppose la dérive casuistique du courant contingent qui considère que chaque entreprise suit son propre cheminement. Dans ce dernier cas, tout serait alors affaire de contexte. Si l'on considère que, pour la plupart de ces travaux, la croissance a été mesurée à partir du critère de taille, une fois de plus, il semble qu'il faille être extrêmement prudent quant aux effets de la taille. Si l'influence de l'évolution de la taille est indéniable, il est tout de même nécessaire d'accepter que les seuils de ruptures entre les différents stades dépendent tout autant de la taille que du contexte dans lequel les entreprises sont en exploitation. Dans le prolongement des propos de Kimberly (1976), il conviendrait alors d'adopter une approche contingente du mode de découpage selon la taille en cherchant sous quelles conditions ce mode pourrait varier. En d'autres termes, les seuils de spécificité sont contingents.

Au total, parce qu'ils accréditent l'idée selon laquelle la taille exerce des changements (effet-taille) et que ces changements sont des différences de nature (métamorphoses), l'ensemble de ces travaux conduit logiquement à faire de la taille un critère pertinent de découpage. En ce sens, les travaux sur la taille et la croissance fournissent les fondements de la recherche en PME, puisque celle-ci repose sur un découpage selon le critère de taille. Toutefois, il convient de préciser les limites de ces fondements. 
- Si l'effet-taille semble unanimement admis, il n'est pas pour autant considéré comme une «loi». Certains effets de la taille peuvent être compensés en tout ou en partie par d'autres variables contingentes. À ce jour, aucune étude n'a prouvé la supériorité du facteur taille sur d'autres facteurs reconnus comme ayant également une influence sur le mode de fonctionnement et de développement de l'entreprise (technologie, environnement, activité, contexte culturel, etc.). Si le rôle de la taille est reconnu de tous, c'est l'importance qu'on lui accorde qui fait l'objet d'un débat. On peut imaginer que dans certains contextes et sous certaines conditions, la taille n'exerce pas ou peu d'effet. Il convient donc de considérer l'effet-taille comme un effet contingent et non universel.

- De plus, aucun modèle de croissance ne fait l'unanimité. Les délimitations selon la taille reposent sur des frontières plutôt floues. Les sauts qualitatifs diffèrent d'une entreprise à l'autre. Par conséquent, si les modèles de métamorphoses sont contingents, il ne saurait exister de frontière critique universellement reconnue. Les seuils de spécificité sont contingents, y compris celui qui sépare le monde de la PME de celui de la grande entreprise.

Tous ces travaux sont parcourus par une opposition classique entre les partisans de l'approche universelle, préoccupés par un souci de généralisation théorique, et les adeptes de la contingence qui, dans un souci de réalisme et de validation empirique, multiplient le nombre de facteurs influents. À l'approche universelle des effets de la taille et des seuils qui en découlent s'oppose une approche plus nuancée qui cherche à définir dans quelle mesure et sous quelles conditions certains résultats sont plus ou moins probables. C'est l'approche contingente. La recherche en PME repose donc sur des postulats plus ou moins contestables. Dans ces conditions, nous verrons comment ce clivage initial va se retrouver dans les travaux ultérieurs concernant la PME.

\section{Les fondements (1975-1985)}

À partir du milieu des années 1970, deux courants distincts, spécialisés dans l'analyse des PME, se développent conjointement. D'une part, le courant de la spécificité a pour projet de mettre en évidence les traits caractéristiques des entreprises de petite taille et de proposer une théorie spécifique de l'organisationPME. La PME est alors considérée comme un objet de recherche. D'autre part, le courant de la diversité cherche à établir des typologies dans le but d'ordonner et de classer l'hétérogénéité du monde des PME. Dans ce cas, la PME est considérée comme un champ de recherche. 


\subsection{Le courant de la spécificité (1975-1985)}

Jusque dans les années 1970, l'objet-PME «n'est pas pris en compte en tant que tel, mais est inclus de fait dans une problématique de gestion globalisante qui gomme le plus souvent toutes ses spécificités » (Bayad et al., 1995). Ce n'est qu'à la fin des années 1970 que la recherche en PME prend un nouvel essor, lorsque plusieurs auteurs ne la considèrent plus comme un modèle réduit de la grande entreprise, mais comme une entreprise à laquelle on peut associer des particularités : la PME est spécifique (Barreyre, 1967 ; Gervais, 1978 ; Dandridge, 1979; Welsh et White, 1981 ; Marchesnay, 1982a, 1982b ; Hertz, 1982, etc.). L'entreprise de petite taille devient « la petite entreprise». La PME se constitue alors progressivement en objet de recherche. Mais en objet de recherche relatif, dans la mesure où la preuve de la spécificité des petites entreprises ne peut se faire qu'à partir d'études comparatives entre les petites, moyennes et grandes entreprises (d'Amboise et Plante, 1987 ; Brytting, 1991). Autrement dit, la thèse de la spécificité de la petite entreprise se nourrit des différences établies comparativement aux grandes entreprises. Nous considérons que l'accumulation et l'intensité des différences mises en évidence entre les petites et les grandes entreprises constituent des signes satisfaisants pour en faire des objets d'une nature différente. Ainsi, Penrose écrit dès 1959: «La croissance a fondamentalement modifié les fonctions de direction et la structure administrative de base, ce qui entraîne une modification profonde de la nature de l'organisme lui-même. Les différences de structure administrative entre les très petites entreprises et les très grandes entreprises sont si importantes qu'à bien des égards, il est difficile de concevoir que les deux espèces appartiennent au même genre. » Enfin, selon Leclerc (1990), « lorsque l'on regarde plus précisément cette entité, on remarque tout d'abord qu'elle n'est appréhendée qu'en termes d'écarts avec les grandes entreprises [...] La PME-PMI ne prend toujours corps que comparativement à la grande entreprise.»

C'est en ce sens que la PME n'est qu'un objet de recherche relatif. L'affirmation de la spécificité des PME n'est pas une thèse en soi mais relative à ce qui la distingue de la grande entreprise. En accordant à la taille la plus grande importance et en considérant que ce facteur occasionne des changements de nature, on peut en déduire que l'entreprise de petite taille est spécifique.

Toutefois, si les travaux concernant le concept de taille fournissent les fondements théoriques sur lesquels la recherche en PME va se développer, le critère de taille sert davantage à opérer des découpages de l'appareil productif qu'à définir des catégories homogènes d'entreprises (Bernard et Ravix, 1988 ; Perrat, 1990). Plusieurs études ont conclu au «caractère arbitraire des frontières assignées $a$ priori dans certaines typologies fondées exclusivement sur la taille » (Candau, 1981). Il faut donc pénétrer la boîte noire. Comme le notent 


\section{FIGURE 1}

\section{Le courant de la spécificité}

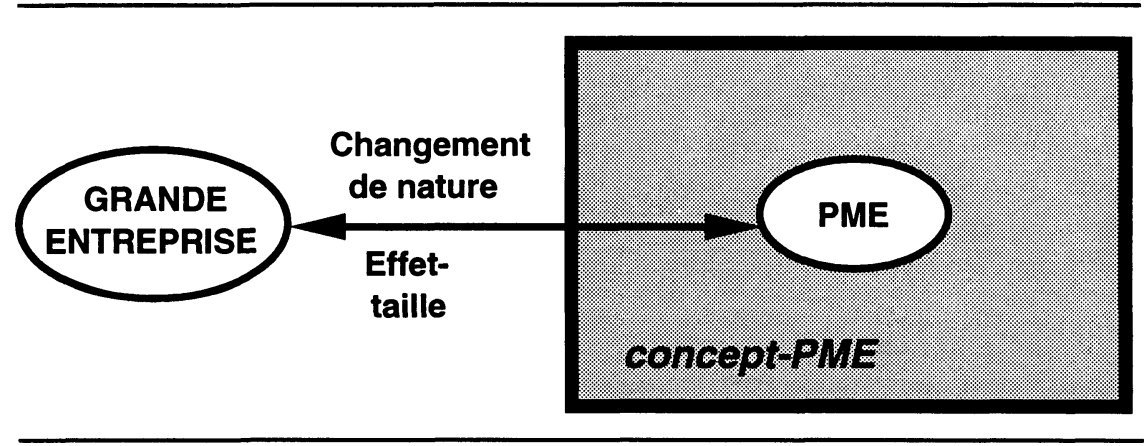

Bayad et al. (1995): "Il est possible de mettre en évidence deux grandes tendances de travaux sur les PME. Elles se définissent schématiquement soit comme la transposition / adaptation d'approches GE en contexte de PME. À l'opposé, des tentatives récentes font abstraction des problématiques classiques de la GE et tentent de jeter un regard neuf sur la question. » C'est la logique d'innovation. L'objectif est alors «[...] de passer d'une phase de vision du phénomène de la petite entreprise, perçue comme institution spécifique d'un capitalisme à une phase de découpage et d'abstraction, où la réalité est conçue autour du type idéal de firme représentative » (Marchesnay, 1982a). Cette étape constitue la phase de conceptualisation de la PME considérée comme un objet de recherche spécifique. Il s'agit généralement de plaider en faveur d'un concept jusqu'alors totalement absent des préoccupations de gestion. Hertz (1982, p. 19) résume bien ce problème dans son ouvrage et plaide, de ce fait, en faveur d'une définition unique et universelle :

L'argumentation à l'encontre d'une définition unitaire et universelle de la petite entreprise tient principalement aux variations quantitatives de la signification de «petite» dans différents pays. Cet argument serait valide s'il était établi que la petite entreprise ne puisse se définir autrement qu'à l'aide de critères quantitatifs de la taille. Mais si l'on accepte que la petite entreprise est un concept et pas simplement une entreprise miniature, alors il devient possible d'en donner une définition uniforme. Un concept doit être défini de façon universelle. Sans définition uniforme de l'objetPME, aucune comparaison ne peut être faite.

Ce courant de recherche porte une attention accrue à la mise en évidence d'uniformités qui résultent des tendances de la petite taille. Malgré l'hétérogénéité du monde des PME, chaque auteur insiste sur les caractéristiques communes. Car ce sont ces invariants qui constituent la base de la spécificité des PME : «Le monde de la PME, considéré individu par individu, se révèle lui-même d'une extrême complexité ; mais pris en tant que tel, des constantes, 
des permanences, des tendances en surgissent à l'examen. »(Julien et Marchesnay, 1988) À partir du milieu des années 1970, partant du constat que la théorie des organisations a été consacrée jusqu'alors exclusivement aux grandes entreprises, plusieurs auteurs déplorent que la spécificité des PME ne soit pas prise en compte par la théorie des firmes (Marchesnay, 1982a) ou les théories des organisations (Gervais, 1978). Il convient donc de proposer de nouvelles théories, de nouveaux cadres d'analyse qui intègrent les particularités de la PME. À cet égard, les intitulés de certains articles sont assez représentatifs du ton revendicatif et engagé de cette période : «Pour une théorie de l'organisation PME » (Gervais, 1978) ; «Pour une taxonomie de l'hypofirme » (Candau, 1981); « Pour une modèle d'hypofirme» (Marchesnay, 1982a). Afin de susciter l'adhésion ou du moins des réactions, les chercheurs en PME avancent arguments sur arguments pour légitimer un courant de recherche qui n'en est qu'à ses balbutiements. "La PME ne peut plus être considérée comme un simple modèle réduit, voire infantile d'un archétype d'entreprise. Tout comme le groupe industriel, elle constitue un être qui a sa propre réalité, sa propre existence. » (Julien et Marchesnay, 1988) Cette évolution de la pensée permet de passer de la «reconnaissance à la connaissance des PME » (Guilhon et Marchesnay, 1994). Dès lors, la tâche n'est plus de découvrir mais de définir ce nouvel objet de recherche. Il s'agit de définir un profil type de la PME en insistant sur les points communs qui caractérisent ce «nouveau monde » scientifique. Cet objet de recherche a reçu diverses appellations : phénomène PME (Hertz, 1982), concept-PME (Julien, 1994), modèle d'hypofirme (Marchesnay, 1982a), idéal type (Julien et Marchesnay, 1992). La quête d'un type idéal repose sur une idée simple : la recherche en PME ne pourra progresser que le jour où la plupart des chercheurs se mettront d'accord sur une définition unitaire et universelle du phénomène PME (Hertz, 1982). Ces propos rejoignent ceux de d'Amboise (1993) qui regrette le caractère fragmenté de la recherche en PME, faute de ne pas disposer d'une définition minimale commune.

De plus, cet effort de modélisation ou de conceptualisation théorique est nécessaire dans le cadre d'une démarche scientifique, car la capacité de généralisation constitue le critère le plus discriminant entre les connaissances scientifiques et les connaissances non scientifiques en gestion comme en d'autres disciplines (Cohen, 1989). Toutefois, si ce courant constitue le point de départ de la recherche en PME, il n'est pas exempt de critiques. En effet, si certains auteurs ont préféré ne retenir que « les constantes, les permanences, les tendances» (Julien et Marchesnay, 1988), si d'autres préconisent une «définition unitaire et universelle » (Hertz, 1982) et si d'autres encore considèrent que dans la PME «il y a plus de déterminisme que de contingence » (Chicha, Julien et Marchesnay, 1990), le risque est qu' « une telle présentation se trouve à "idéaliser" l'idéal type » même si «elle reflète des tendances observées au-delà de l'inévitable normativité des auteurs» (Marchesnay et 
Julien, 1992). Au souci légitime de généralisation succède souvent une sorte d'universalisme de la spécificité.

L'ouvrage récent de Bauer (1993) constitue un exemple parfait des excès de ce courant. Conscient de la nécessité d'insister sur les particularités de la PME, Bauer n'hésite pas à expliciter cette nature autour d'une loi fondamentale : «Au nom du Père, du fils et de l'entreprise. » «Nous avons pu montrer qu'un patron de PME agit selon une triple logique : une logique économique, une logique politique et une logique familiale. Cette loi fondamentale permet de caractériser son activité professionnelle, c'est-à-dire l'ensemble des décisions qu'il prend dans sa firme comme produit d'une triple rationalité.» (Bauer, 1995) Même si, comme nous le verrons par la suite, Bauer atténue ses propos, l'évocation d'une loi atteste de la dérive dogmatique qui affecte fréquemment le courant de la PME-objet. À trop vouloir mettre en relief les spécificités liées à la petite taille, les auteurs sont enclins à tenir pour lois universelles ce qui n'est que tendances probables.

En général, les chercheurs en PME se contentent de constituer un échantillon d'entreprises de petite taille pour pouvoir faire référence au corpus théorique de la recherche en PME. Ce procédé a la vertu d'une grande simplicité, mais il repose sur de forts présupposés qui sont loin d'être toujours vérifiés. Le passage du concept de petite taille au concept de PME n'est pas automatique. Or, la plupart des chercheurs font « comme si » ce lien était parfait, comme s'il suffisait d'avoir une entreprise correctement définie en termes de petite taille pour faire le lien avec le concept de PME. Selon nous, deux types d'erreurs peuvent être commises lors de cette étape. Une erreur de première espèce consisterait à accepter à tort l'hypothèse de la spécificité de la PME auprès d'entreprises qui, malgré leur petite taille, ne le sont pas. Une erreur de deuxième espèce serait de rejeter à tort, du cadre de validité du concept-PME, des entreprises sous prétexte qu'elles sont de grande taille. Or, comme l'ont montré Bournois et Pellegrin (1994), une entreprise de grande taille peut conserver les caractéristiques organisationnelles propres au concept de PME. La portée du concept de PME est donc à la fois plus large et plus étroite qu'il n'y paraît. Plus large, car des entreprises de grande taille peuvent être analysées avec profit à partir du cadre théorique du concept-PME. Plus étroite, car toutes les entreprises de petite taille ne rentrent pas forcément dans ce cadre. Pour éviter ces types d'erreur, il conviendrait de vérifier l'existence empirique des caractéristiques théoriques du concept-PME. Nous employons à dessein le conditionnel car, à notre connaissance, cette précaution est très rarement prise. En d'autres termes, si «le » critère de sélection des entreprises d'un échantillon est généralement la taille, les caractéristiques qualitatives, à l'exception du critère d'indépendance juridique, ne sont jamais vérifiées mais supposées empiriquement. La thèse de la spécificité de la PME est alors un postulat sur 
lequel chaque chercheur se fonde sans en vérifier l'existence empirique. Certes, nous ne nions pas la tendance selon laquelle plus la taille est petite, plus le rôle du dirigeant peut prendre de l'importance. Mais cette tendance fait ressortir une caractéristique qui relève plus du probable que de la certitude. Pourtant, dès lors que l'on travaille sur les PME, cette caractéristique est constamment mise en avant pour légitimer son intérêt prioritaire pour les objectifs et le comportement du dirigeant. La relation petite taille-prépondérance du rôle du dirigeant est infaillible à un point tel que, pour Bayad et Nebenhaus (1994), le rôle du dirigeant fait partie «des idées obligatoires dans tout travail théorique sur les $\mathrm{PME}$ ». Ce type de démarche ne peut conduire qu'à surestimer le rôle et l'importance de la taille. Dans ce cas, la théorie PME s'apparente à une théorie universelle.

Au total, si la thèse de la spécificité présente une faible autonomie de recherche du fait des nécessaires comparaisons qui doivent être faites pour étayer la preuve de sa spécificité, elle se caractérise par un fort degré de généralisation puisqu'il s'agit de rompre avec la vision traditionnelle de la PME (grande entreprise miniature) pour proposer de nouvelles bases théoriques aux futurs travaux sur la PME. Toutefois, comme le note Léo (1987):

Il n'est sans doute pas exact de considérer la PMI comme un modèle d'entreprise systématiquement opposable à la grande entreprise. La frontière entre les deux types est floue et difficile à fixer; tout ce que l'on peut observer sur les PMI n'est pas fait en dehors de l'influence des grandes entreprises qui ont leur part de responsabilité dans ce que sont les PMI aujourd'hui (liens de complémentarité, transfert de technologies, sous-traitance...) Mais surtout cette opposition PMI-Grande Entreprise, révélatrice à certains points de vue, n'est plus féconde dès que l'on approfondit l'analyse : ce qui domine l'univers des PMI, c'est l'hétérogénéité [...] Cette diversité est une des dimensions mêmes du phénomène PMI qui peut expliquer une partie de son dynamisme actuel.

Dans ces conditions, la thèse de la spécificité peut s'avérer trop réductrice de la variété des PME. En d'autres termes, si la thèse de la spécificité a une vertu homogénéisante, elle s'accommode mal de l'extrême hétérogénéité qui semble caractériser le monde des PME. Ainsi, Candau, dès 1981, considérait que

[...] au fur et à mesure du développement des connaissances sur les petites entreprises, la variété de leurs caractéristiques paraît devoir largement l'emporter sur leur uniformité. De ce fait, une contribution importante serait apportée en étudiant empiriquement la diversité des formes adoptées par les entreprises, et en les réduisant en un nombre limité de classes ayant en commun des caractéristiques relativement uniformes. Ceci serait beaucoup plus significatif que d'essayer de formuler des lois prétendant s'appliquer à toutes les organisations, pourtant susceptibles de constituer des univers séparés, ou d'élaborer des typologies a priori, normatives, ayant un faible pouvoir explicatif. 
Ces propos rejoignent ceux de Bernard et Ravix (1988) pour lesquels la recherche de l'hétérogénéité a été particulièrement active dans le sous-ensemble des PME:

La limite fondamentale de l'analyse du système productif à l'aide du découpage par taille vient du fait qu'elle définit des sous-ensembles réputés homogènes d'entreprises ; cette propriété, qui est une vertu statistique, est cependant un obstacle à une connaissance de la diversité des unités de production. La solution à ce problème d'homogénéité a été de s'appuyer sur des études d'échantillons d'entreprises, dans le but de mettre en évidence des spécificités de structures et de comportements au sein même des catégories de taille.

Aussi, parallèlement aux tentatives de généralisations théoriques du courant de la PME-objet, plusieurs travaux empiriques vont être menés dans le but de mieux cerner la diversité du champ des PME en dressant des typologies de firmes à l'intérieur des classes de taille.

\subsection{Le courant de la diversité (1975-1985)}

On a très souvent tendance à considérer les PME comme un bloc homogène dès lors qu'on les oppose aux grandes entreprises. Cette distinction est d'autant plus valable que la comparaison se cantonne à ne retenir que les situations extrêmes. Mais lorsqu'on s'intéresse uniquement aux entreprises de petite taille, il semble difficile, voire impossible de les regrouper autour d'un modèle unique. Du fait de la diversité du champ des PME (PME-Champ), aucune généralisation n'est possible et tout est alors affaire de contexte. Les PME sont considérées comme un ensemble trop hétérogène pour se prêter à une tentative de généralisation. $\mathrm{Si}$ le courant de la spécificité considère que «plus la taille de l'entreprise est petite, plus cette dernière est spécifique », il ne s'agit que d'une tendance. Or, comme le note March (1994) : «Tout essai de description des tendances de gestion, même s'il est valide en moyenne, ne décrit de façon adéquate aucune situation de gestion particulière. » Dans ces conditions, la PME n'est pas une catégorie homogène, mais une appellation commode qui désigne une réalité multiple susceptible de se différencier par l'activité, par la forme de propriété, par les stratégies adoptées, par les modes de gestion, etc., « on sait qu'il est difficile de parler d'une théorie des PME alors que celles-ci sont extrêmement hétérogènes [...] on ne peut donc échapper à une approche de contingence. » (Julien, 1994). De même, pour Bayad et Nebenhaus (1994), «contrairement aux Grandes Entreprises, pour les PME il est difficile de mettre en évidence des invariants de gestion ». Enfin, pour Mahé de Boislandelle (1994), « la démarche de théorisation est difficile et périlleuse, car il s'agit surtout de saisir la diversité et le contingent ». La proposition de Mahé de Boislandelle se situe aux antipodes du plaidoyer de Hertz en faveur d'une définition unique et universelle du concept-PME. 
Dans ces conditions, il convient d'identifier l'ensemble des facteurs de contingence qui exercent un effet sur la nature de l'organisation. Prenons un exemple particulièrement illustratif de ce type d'approche. Dans l'optique d'une démarche contingentielle, Fabi, Garand et Pettersen (1993) établissent une liste des différents facteurs qui exercent une influence sur la gestion des ressources humaines (GRH) en cadre PME. Partant d'un recensement exhaustif, depuis les années 1950 jusqu'à la fin des années 1980, de la littérature concernant ce thème précis (au total, 75 études empiriques), ils recensent 21 facteurs de contingence différents qu'ils regroupent au sein d'un modèle contingentiel de la GRH en PME. Cette compilation montre clairement la diversité et la dispersion des variables retenues par les chercheurs pour expliquer les phénomènes liés aux pratiques de GRH en PME. Ce modèle constitue une bonne grille d'analyse, destinée à inventorier les pratiques en matière de GRH, en incluant les variables contingentes susceptibles d'exercer une influence. La valeur d'un tel modèle est essentiellement heuristique. Dans une démarche d'intervention ou de conseil en entreprise, ce type de modèle est souhaitable et utile. Il s'efforce de serrer au plus près les pratiques effectives de la GRH en PME.

Mais, relativement à notre propos, ce modèle illustre les excès de l'approche contingente. En effet, ce qu'il gagne en descriptivité, il le perd en prédictivité. La multiplicité des facteurs pris en compte constitue un frein à l'élaboration d'un cadre de référence général. En effet, sur la base de 21 facteurs de contingence et en supposant que chaque facteur présente deux modalités (hypothèse minimale), le nombre de situations différentes possibles est de l'ordre de 2097 152. S'il est légitime que les auteurs cherchent à rendre compte de l'hétérogénéité des entreprises de petite taille, le problème est que le nombre de situations théoriquement envisageables par le jeu des combinaisons entre les divers facteurs dépasse la diversité réelle des entreprises. À l'irréalisme des modèles universels s'oppose le surréalisme des approches contingentes. Poussée jusqu'à l'extrême, l'approche contingente tend à considérer chaque entreprise comme un cas unique. Il devient impossible de généraliser et de proposer une théorie de la PME. Nous qualifions ce type d'excès, de dérive casuistique.

Aussi, afin de ne pas multiplier inutilement le nombre de combinaisons différentes, la solution consiste soit à se limiter aux facteurs les plus influents en dressant des typologies se limitant à un aspect particulier, soit à considérer que certaines combinaisons sont plus probables que d'autres (hypothèse de congruence). La recherche de typologies fondées exclusivement sur des échantillons de PME a été particulièrement active entre 1975 et 1985 (Bernard et Ravix, 1988 ; Julien, 1994). Les différents types établis retracent alors la diversité exclusivement au sein des PME. «Les profils de PMI permettent d'intégrer dans l'analyse la diversité de leurs comportements. » (Léo, 1987) 


\section{FIGURE 2}

\section{Le courant de la diversité}

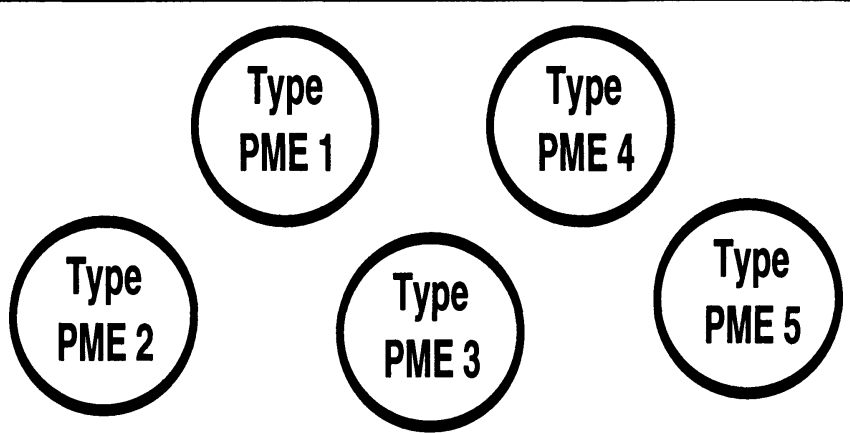

Le courant de la diversité présente les caractéristiques inverses de celui de la spécificité.

- Parce que les échantillons sont construits exclusivement à partir d'entreprises de faible dimension, le courant de la diversité présente une forte autonomie de recherche par rapport aux travaux sur la grande entreprise. Il ne s'agit plus de comparer la petite entreprise à la grande, mais d'évaluer, dans l'absolu, la diversité de ce monde particulier.

- Cependant, la multiplicité des types établis ne permet pas de faire émerger un cadre général, soit parce qu'il y en a plusieurs (approche typologique), soit parce qu'il y en a une infinité (approche contingente). Les résultats obtenus présentent donc un faible degré de généralisation.

En définitive, la recherche en PME peut schématiquement se décomposer en deux courants distincts, car antagonistes tant en ce qui concerne les objectifs qu'ils se fixent que les approches qu'ils préconisent :

1. Le courant de la spécificité s'efforce de définir l'objet-PME considéré comme une institution singulière. La démarche est volontairement universelle et unitaire puisqu'il s'agit de préciser les caractéristiques propres à la PME (les spécificités) et d'en déduire des problématiques de recherche ad hoc tout en facilitant l'accumulation des connaissances sur la PME. Ce courant court le risque de dérive dogmatique, car il cherche à tout prix à généraliser un modèle (PME-Objet), ce qui conduit insidieusement à l'idée que toutes les PME sont spécifiques et conformes au modèle théorique.

2. Le courant de la diversité s'efforce de simplifier la diversité du champPME en dressant des types distincts. Cette orientation se situe aux 
antipodes de la thèse de la spécificité. L'approche est beaucoup plus contingente et la portée théorique plus limitée. Cette approche est plus réaliste, mais limite les connaissances de la PME au niveau d'énoncés anecdotiques. Sous couvert de réalisme, on en déduit qu'aucune généralisation n'est possible et que tout est alors affaire de contexte. C'est le risque de dérive casuistique.

L'homogénéité de l'objet-PME semble en contradiction avec l'hétérogénéité du champ-PME. La distinction entre PME-champ et PME-objet n'est donc pas neutre sur le plan théorique. Comme nous en faisions l'hypothèse, le clivage entre universalisme et contingence relevé dans les travaux concernant la taille et les modèles de croissance traverse également les travaux concernant la PME. C'est en accordant à la taille la plus grande importance (effet-taille absolu) et en supposant que les changements de nature occasionnés par la taille sont communs à tous les types d'entreprises (modèle de métamorphose universel) que toutes les PME peuvent être considérées comme spécifiques. L'universalisme du courant de la spécificité (1975-1985) découle de l'universalisme des courants de l'effet-taille et des modèles de métamorphoses (19651975). Mais des prolongements récents (1975-1990) tendent à montrer que l'effet-taille est contingent et que les seuils critiques ne sont pas clairement identifiables (les modèles de métamorphose sont divers). D'après les résultats de ces travaux, l'analyse des PME devrait être contingente et intégrer l'hétérogénéité. Cette approche correspond pleinement à celle du courant de la diversité.

Tout chercheur en PME doit se positionner par rapport à ces différents courants, car les problématiques de recherche ne sont pas les mêmes. À l'approche universelle du courant de la spécificité s'oppose donc l'approche contingente du courant de la diversité. Marchesnay (dans Julien et Marchesnay, 1988) qualifie cet antagonisme de véritable «dilemme» devant lequel le chercheur en PME se trouve placé :

- Rechercher des types idéaux, des catégories holistes mais se heurter dans ces conditions au risque de non-pertinence empirique.

- S'efforcer de serrer au plus près les comportements effectifs et établir des typologies en fonction de chaque problème étudié. Mais dans ces conditions le risque est grand de multiplier les typologies sans bénéficier d'un cadre de référence général [...] Ce que gagnent de telles constructions en descriptivité, elles le perdent en prédictivité, comme il l'a souvent été reproché aux approches contingentes.

Comment alors concilier ces deux courants de recherche ? Comment rendre compte tout à la fois de la singularité et de la diversité de ces objets particuliers que sont les PME ? En somme, comment répondre à ce que Julien et Marchesnay (1992) qualifient de «problématique complexe » ? C'est dans 
cette perspective que s'inscrivent les prolongements de la recherche en PME à partir du milieu des années 1980.

\section{Les prolongements (1985-1995)}

En croisant les antagonismes de la recherche en PME (universalisme contre contingence et spécificité contre diversité), on obtient une matrice à quatre cases qui fait ressortir deux voies de prolongements envisageables (quadrants A et B).

GRAPHIQUE 1

Les antagonismes de la recherche en PME

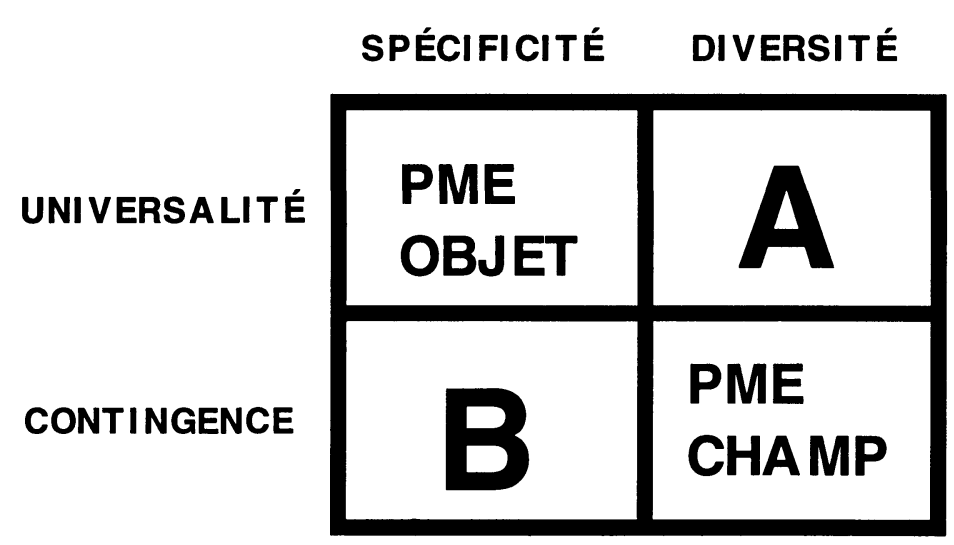

La première cherche à concilier la diversité dans l'universalité de la spécificité de la PME. C'est le courant de la synthèse. Mais, un autre courant considère, de façon plus ou moins explicite, que la spécificité de la PME est contingente à un cadre de validité. Pour délimiter les frontières de cette spécificité, il convient alors d'identifier des contextes dénaturant la PME. C'est le courant de la dénaturation.

\subsection{Le courant de la synthèse (milieu des années 1980)}

Cette prise de conscience de la nécessité d'intégrer la diversité aux travaux concernant la spécificité prend un réel essor à la fin des années 1980, même si certains travaux antérieurs à cette période avaient déjà insisté sur ce point. La particularité de ce courant est de considérer que la spécificité du concept-PME est modulable, c'est-à-dire que les PME sont plus ou moins spécifiques. Les caractéristiques spécifiques de la PME ne sont pas strictes mais élastiques. Ainsi, 
Julien (1994) dans $P M E$ : bilan et perspectives propose une «typologie sur continuum » qui peut s'apparenter à une sorte de polymorphisme de la spécificité de la PE. Cette «synthèse spécificité / diversité » présente l'avantage d'intégrer la diversité des PME tout en gardant intacte la spécificité de la PME, c'est-à-dire l'essence de l'objet de recherche. Il y a une diversité au sein de la spécificité. De même, Bauer (1995), après avoir énoncé la loi fondamentale qui caractérise les PME, poursuit son analyse en considérant que

[...] la prise en compte des logiques d'action économiques, politiques et familiales, permet de construire des typologies exprimant la grande diversité des patrons de PME et leur firme, des situations au sommet des PME comme des actions qui y sont menées. Caractériser un patron de PME par la taille relative de chacune de ses trois têtes conduit à imaginer un nombre infini d'éventualités, situées toutes dans un espace à trois dimensions. Pour cerner les traits essentiels d'un patron de PME, il faut donc le situer dans cet espace à trois dimensions; et ce n'est que dans un deuxième temps qu'il convient d'affiner l'analyse et de dessiner plus précisément les traits de ses têtes les plus développées.

L'ouvrage de Bauer constitue une synthèse entre la particularité des PME et leur diversité. Une fois de plus, les traits spécifiques de la PME (dans ce cas, la loi fondamentale) constituent un contour plus ou moins large au sein duquel une grande variété de cas différents peuvent s'insérer. La loi énoncée par Bauer est donc un cadre d'analyse permettant de situer la diversité du monde des PME à partir d'une logique qui met en relief les spécificités de l'objet étudié. Si Julien (1994) considère que l'on ne peut échapper à une approche contingente du fait de l'hétérogénéité des PME, il rajoute que cette contingence n'empêche pas pour autant de retrouver des similitudes suffisantes pour constituer une nouvelle théorie économique et de gestion pour les PME : «en tenant compte des comportements des PME dans la turbulence de l'économie locale et nationale, on peut dépasser la simple contingence pour trouver certaines généralisations susceptibles de nous conduire à une théorie analytique sinon prédictible pour justifier la renaissance de ces unités de production dans nos économies ».

La prise en compte de la diversité par les tenants de la thèse de la spécificité des PME débouche sur une évolution significative de la pensée en PME. Il ne s'agit plus de dresser un profil type de LA PME prise comme un objet théorique, mais plutôt de rechercher des cadres d'analyse ou des modèles heuristiques capables de retranscrire la diversité du monde réel. Cet avis rejoint celui de Marchesnay (1991):

Les milieux de PE sont d'une effroyable complexité : d'où de la part du chercheur la tentation du réductionnisme, rapidement vouée à l'échec. Il faut alors se rabattre sur des grilles d'analyses heuristiques, évoquer des configurations, au sens de Mintzberg, proposer des conjectures à partir de construits et de variables réfutables, soit par la validation logique, soit par la validation empirique. C'est dans cet esprit que nous proposons une théorie de l'hypofirme. 
Au total, si en première analyse la PME peut être synthétisée autour d'un idéal type, rapidement les modèles initiaux vont s'avérer trop réducteurs de l'extrême hétérogénéité de ce monde particulier. La recherche en PME s'oriente alors vers ce que Martinet (1986) appelle une Forme : « La forme, invariante pour un temps, ne recouvre pas une réalité intangible et délimitée. Elle rend compte, en les accueillant, des modulations concrètes. En ce sens, elle est davantage conceptacle que concept. Elle peut tolérer l'ambiguïté. » Tandis que le concept délimite, découpe et disjoint, la forme polarise, dessine, agglomère. Pour Durand (dans Martinet, 1986), la forme a essentiellement une valeur heuristique : en tant que telle, elle n'existe pas ; c'est un ensemble vide mais qui permet de comprendre les apparences existantes. Le concept-PME devient alors plus flou, plus vague, plus imprécis. Étant donné la nature profondément hétérogène des $\mathrm{PME}$, le concept-PME prend les traits d'une forme. Dans cette optique, plusieurs auteurs établissent des cadres d'analyse au sein desquels il est possible d'établir des typologies, lesquelles découlent de la prise en compte préalable de certains traits spécifiques aux PME. Il s'agit de typologies ad hoc.

\section{FIGURE 3}

\section{Le courant de la synthèse}

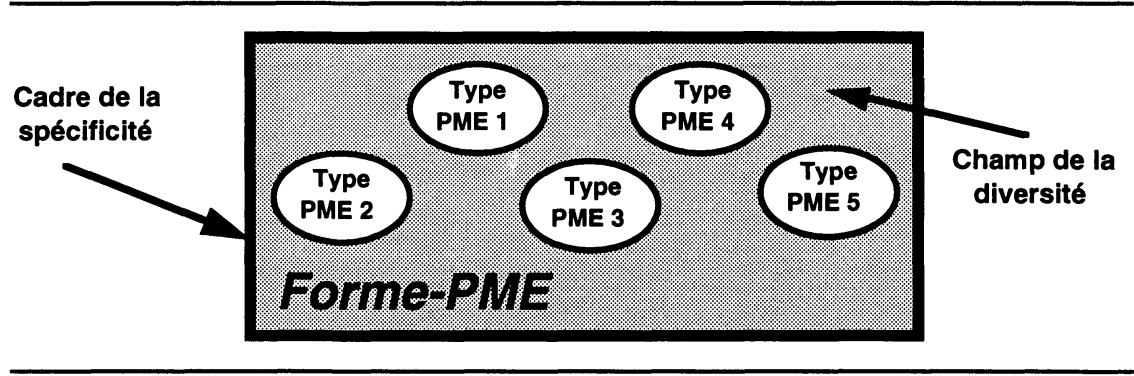

Cette voie a déjà été évoquée dès 1981 par Candau qui, dans son plaidoyer «pour une taxonomie de l'hypofirme», affirme qu' «il est impossible d'étudier les PME, abstraction faite de la personne du chef d'entreprise, de ses motivations et de sa famille qui sont les facteurs caractéristiques dominants de l'hypofirme ». Toutefois, il reconnaît qu'il serait vain de vouloir formuler des lois prétendant s'appliquer à toutes les organisations, pourtant susceptibles de constituer des univers séparés. «La multiplicité des caractéristiques spécifiques des petites entreprises interdit a priori de les assimiler à un ensemble homogène [...] La variété de leurs caractéristiques paraît devoir largement l'emporter sur leur uniformité. » Candau (1981) met donc en relief une des particularités des PME (le rôle du dirigeant) au point d'en faire le centre de sa démarche de recherche. En effet, si l'auteur s'intéresse à la structure administrative 
des entreprises, c'est précisément parce qu'il suppose que c'est par ce biais que vont se manifester les motivations, la rationalité et les conceptions du dirigeant. «La réintroduction de la personne du chef d'entreprise dans l'étude des hypofirmes entraîne celle de ses rôles qui ne fait que traduire sa logique d'action, c'est-à-dire sa forme de rationalité et les conceptions qu'il va adopter dans la conduite de son entreprise. » La personnalisation du mode de gestion des PME est bien le postulat de départ sur lequel Candau s'appuie pour établir sa taxonomie. Malgré l'hétérogénéité qui caractérise l'univers des PME, il considère que l'importance du rôle du dirigeant est l'invariant fondamental qui participe à définir la spécificité des PME par opposition aux grandes entreprises. En ce sens, le modèle d'hypofirme sert de modèle théorique de base à partir duquel il identifie la ou les variables pertinentes. La recherche est fondée sur les spécificités reconnues aux PME tout en mettant en relief la diversité de cas. Les travaux de Candau (1981) constituent bien une jonction entre les deux courants de recherche qui se développent jusqu'alors parallèlement. Ils tiennent compte conjointement de la spécificité et de la diversité des PME.

Toutefois, cette voie de synthèse, même si elle présente un progrès, reste toujours universelle. En effet, ces recherches partent du point qu'il s'agit de démontrer, à savoir le rôle de la forme-PME dans le fonctionnement des entreprises de petite taille. Nous ne nions pas que ce lien existe. Nous contestons qu'il faille, pour en rendre compte, partir d'une problématique qui le présuppose. Combien de chercheurs postulent la spécificité de la PME pour justifier, par exemple, qu'ils s'intéresseront au rôle du dirigeant et pour montrer lors de l'étude empirique qu'effectivement le dirigeant joue un grand rôle ? Les problématiques partent en effet d'une définition normative de la petite entreprise (l'objet-PME), énonce ce qu'une entreprise de petite taille doit réunir pour être qualifiée comme telle, puis en observent l'existence empirique, soulignent la pluralité des formes, dressent des typologies. Par conséquent, ce type de démarche ne rencontre jamais que ce qu'elle a présupposé, à savoir la spécificité de l'objet-PME. En d'autres termes, il ne faut pas oublier que l'entreprise de petite taille peut relever théoriquement d'un mode de fonctionnement qui ne correspond pas à cet objet ${ }^{2}$. La thèse de la spécificité est utilisée comme un point de départ (un postulat) duquel on déduit des hypothèses de travail ad hoc. Or, une entreprise de petite taille peut ne pas être conforme au concept-PME. Si l'on admet l'idée que la PME puisse avoir une nature propre, nous devons admettre son corollaire: sa dénaturation. Une PME peut parfois ne pas ou ne

2. Nous reproduisons, mutatis mutandis, la même argumentation formulée par A. Rallet à propos des problématiques concernant le rôle de la proximité dans le processus d'innovation dans "Choix de proximité et processus d'innovation technologique », Revue d'Économie régionale et urbaine, $\mathrm{n}^{\circ}$ 3, p. 365-386, 1993. 
plus être spécifique. Même si la typologie sur continuum permet de tenir compte de l'hétérogénéité du monde des PME, il est clair qu'implicitement toutes ces typologies présentent un point commun : la spécificité d'un mode de fonctionnement de l'entreprise qui s'apparente à la forme-PME. En d'autres termes, la diversité des types de PME ne dépasse jamais le cadre de la spécificité. De sorte que, malgré la prise en compte de la diversité, le courant de la synthèse s'inscrit dans la lignée de l'approche universelle qui traverse la recherche en PME depuis ses débuts.

Aussi, cette voie de synthèse peut être considérée comme universelle. La thèse de la spécificité n'est jamais remise en cause, elle est simplement modulée. La diversité ne signifie que des changements de degré au sein du cadre universel de la spécificité. C'est la raison pour laquelle on positionne ce courant dans le quadrant A. Or, les changements de degré ne peuvent-ils pas s'accompagner de changements de nature?

\subsection{Le courant de la dénaturation (milieu des années 1990)}

Poser cette question conduit à ne plus considérer la thèse de la spécificité comme un postulat, mais comme une simple hypothèse de recherche réfutable. Il ne s'agit plus d'ériger le dogme de la spécificité comme le cadre absolu de la recherche en PME mais d'entamer un examen critique de cette thèse. Jusqu'à quel point le cadre d'analyse proposé par les chercheurs en PME est-il valide ? La question n'est pas de savoir si le concept-PME est pertinent ou non, mais de délimiter son champ de validité. Dans quelles conditions peut-on accepter la thèse de la spécificité et à partir de quel moment ce concept n'est-il plus valide ? Pour que l'objet-PME puisse être un véritable objet de recherche, il faut non seulement le définir, mais aussi en fixer les limites. Il convient donc d'adopter une démarche contingente (réfutable) de la spécificité.

La figure 4 illustre notre propos. Il ne s'agit pas de remettre en cause la thèse de la spécificité, mais simplement d'adopter une démarche critique, nécessairement contingente, appliquée à l'objet-PME. Existe-t-il des contextes où les entreprises de petite taille ne sont plus conformes à la forme-PME (le type 5 sur notre schéma)? En d'autres termes, quels sont les contextes qui dénaturent la PME ? Ainsi, la définition de la PME énoncée par Guilhon, Guilhon et Peguin (1995) correspond avec justesse à cette évolution de la pensée en recherche PME. Partant de l'idée que l'internationalisation constitue un «choc » qui implique de profonds changements dans le mode de fonctionnement de l'entreprise, surtout lorsque celle-ci est de faible dimension, les auteurs considèrent qu'il existe une contradiction entre l'activité d'exportation et «l'identité de la PME» (Ibid., 1993). Afin de montrer cette hypothèse de travail, ils introduisent dans leur définition de la PME, le concept de contrôlabilité 
Figure 4

Le courant de la dénaturation

(diversité et contingence de la spécificité)

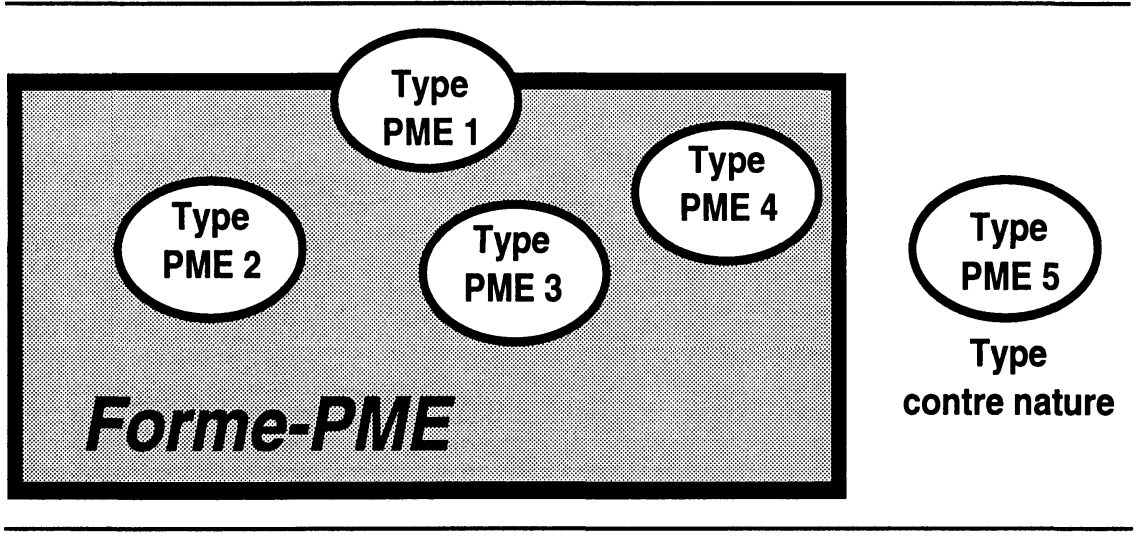

qu'ils définissent « comme la capacité de garder la maîtrise de la mise en œuvre et du développement d'un ensemble de ressources ». La notion de contrôlabilité se situe au cœur de la définition proposée : «Les PME sont définies à partir de cette notion de contrôlabilité qui devient le pivot des différentes parties de leur structure organisationnelle. » L'intérêt d'une telle définition est qu'elle évoque le risque de perte de contrôlabilité et autorise donc, selon nous, l'existence de situations contre nature à la forme-PME : « un développement déséquilibré de la PME est susceptible de remettre en cause son existence propre en tant que PME » (Guilhon et al., 1995). Une entreprise peut ne plus correspondre à la forme-PME standard tout en demeurant de petite taille. Cette définition récuse donc tout universalisme puisqu'elle offre la possibilité d'identifier les limites (contingences) de la forme-PME.

Notons que le souci de délimiter le concept de PME n'est pas nouveau. En 1967, Barreyre intitule sa thèse de doctorat «L'horizon économique des petites et moyennes entreprises ». L'auteur rappelle dès l'introduction que l'origine étymologique hellénique du mot « horizon » contient dans son essence même l'idée de limite. La définition de Barreyre fournit bien les limites du concept-PME puisque sont exclues, d'une part, les entreprises qui, malgré leur petite taille, sont sous l'emprise d'un groupe et, d'autre part, les entreprises qui, malgré leur autonomie réelle de financement et de gestion, ne satisfont pas au moins à cinq des neuf critères associés au concept de PME. Autrement dit, en suivant les propos de Barreyre (1967), on peut en déduire que la constitution d'un échantillon d'entreprises indépendantes et de petite taille est une condition nécessaire, mais pas suffisante pour s'inscrire dans le cadre du paradigme de la spécificité de la PME. Il faut en plus veiller à ce que la majorité des autres 
caractéristiques du concept-PME soit vérifiée. On ne peut donc pas se contenter de construire un échantillon sur la seule base de critères quantitatifs, censés mesurer le concept de taille, pour pouvoir faire référence au corpus théorique concernant l'objet-PME. Par l'énoncé de cette règle, Barreyre fait allusion, sans toutefois l'évoquer explicitement, à l'idée d'une dénaturation possible de la PME.

Après avoir identifié des types «contre nature » (réfutation empirique), le travail consisterait alors à proposer une reformulation de la spécificité de la PME. Il s'agirait d'expliquer pourquoi la PME, placée dans un tel contexte, n'est plus conforme au modèle général (réfutation logique). Ce type de démarche est, selon Dubin (dans Desreumaux, 1992), la démarche à suivre pour la construction d'un modèle théorique, lequel doit respecter un certain nombre de conditions élémentaires, à savoir :

- condition 1 : énoncé des variables pertinentes du système étudié ;

- condition 2: spécification des lois de relations entre ces variables;

- condition 3 : description des états résultant du système ;

- condition 4 : délimitation des frontières ou limites de validité de ces lois.

Si la recherche en PME a fortement progressé sur les trois premiers points, force est de constater que le quatrième point reste encore peu développé. Néanmoins, depuis le début des années 1990 , on peut relever quelques contributions desquelles émerge l'idée d'une dénaturation de la PME autrement que par la croissance et la taille de l'entreprise. Cela signifie alors que la frontière critique qui sépare les mondes des PME et des grandes entreprises n'est pas fondée sur le seul critère de la taille. Ces travaux semblent récuser l'universalité de la spécificité de la PME. Prenons quelques exemples.

\subsubsection{Dénaturation de la PME et autonomie de gestion}

Curvalle (1994) dans une communication intitulée «L'EDI et le JAT condamnentelles les PME? » s'interroge sur l'impact des méthodes de juste-à-temps et d'échange de données informatisées sur le mode de fonctionnement des PME. De même, dans une perspective précisément contingente, Dubost (1995), tout aussi interrogative se demande si « les PME françaises évoluent vers des bureaucraties », c'est-à-dire qu'elles s'éloigneraient du modèle traditionnel de la PME du fait de l'augmentation des phénomènes de contrôle externe et de dépendance dont elles font l'objet. De même, Bayad et Nebenhaus (1994) s'inquiètent pour «l'avenir des PME qui, tout en restant [...] indépendantes, juridiquement parlant, se verront contraintes de se soumettre à des normes extrêmement contraignantes au plan de la gestion, de l'organisation et en particulier en GRH [...] » Ils font 
alors référence aux PME qui, pour conserver leurs marchés de sous-traitance, sont amenées à appliquer les principes issus des normes ISO 9000 . Au total, si l'indépendance juridique est depuis longtemps utilisée comme un critère apte à définir l'appartenance ou pas d'une entreprise de petite taille au monde des PME, la notion d'indépendance organisationnelle (plus difficile à cerner que l'indépendance juridique) paraît devoir jouer le même rôle. Le modèle-PME serait-il un modèle d'autonomie de gestion?

\subsubsection{Dénaturation de la PME et indépendance financière}

En ce qui concerne les activités financières de l'entreprise, plusieurs études tendent à montrer que les caractéristiques traditionnelles (spécificités) des PME sont sensibles aux modes de financement. L'ouverture du capital soit par capitalrisque (Stephany, 1993), soit lors d'une introduction sur le second marché (Belletante et Desroches, 1994) tend à réduire les spécificités des PME, voire à les remettre en cause comme cela peut être le cas lors d'un rachat d'une PME par un groupe. En interprétant ces résultats selon l'optique qui est la nôtre, la question est de savoir où placer le seuil critique délimitant la spécificité du modèle-PME. Entre l'indépendance financière totale où l'entrepreneur finance lui-même tous ces investissements et la dépendance financière totale où l'entreprise devient la filiale d'un groupe et, par voie de conséquence, est exclue du monde des PME, il existe plusieurs situations intermédiaires où les caractéristiques spécifiques de la PME s'atténuent plus ou moins fortement. On peut alors proposer deux seuils. Dans une optique restrictive, on considérera que le modèlePME est un modèle d'indépendance financière. Partant de là, toute autre situation est dénaturante. Ainsi, une entreprise de petite taille qui fait appel à une société de capital de risque ou qui émet des actions n'est plus une PME au sens théorique. Mais on peut avoir une définition plus large et considérer que le modèle-PME est un modèle de non-dépendance financière. Dans ces conditions, seules les PME appartenant à un groupe sont exclues. Cette deuxième conception paraît largement majoritaire au sein de la communauté scientifique en PME. Mais le développement des pratiques de capital de risque et la création de marchés des capitaux plus adaptés aux entreprises de petite et moyenne taille comme le second marché et le hors-cote ne sont-elles pas des tendances récentes de nature à transformer radicalement les modes de financement traditionnels de la PME et, par voie de conséquence, à dénaturer le modèle-PME tout entier?

Outre leur aspect récent, ces travaux portent sur des tendances et des phénomènes relativement nouveaux pour les PME (EDI, JAT, contrôle externe, ouverture du capital, etc.) Leur particularité (et point commun) est de montrer dans quelle mesure certains contextes ou certaines évolutions rendent le conceptPME caduc ou susceptible de profondes mutations. Ces travaux s'inscrivent 
pleinement dans la phase d'examen critique de la conceptualisation de l'objetPME. C'est la raison pour laquelle nous les regroupons dans le courant de la dénaturation. De plus, ils confortent l'idée d'une contingence de la spécificité de la PME.

Contrairement au courant de la spécificité, le courant de la dénaturation ne se fonde pas sur un clivage selon la taille. La référence à la grande entreprise n'est plus nécessaire. En effet, il s'agit de s'interroger sur l'identité de la PME, non par rapport à une classification selon la taille, mais par rapport à ses caractéristiques propres (Guilhon et al., 1993). Dans ces conditions, la PME se constitue en objet de recherche absolu. De plus, la mise en évidence de contextes incompatibles au concept-PME ne rend pas pour autant ce dernier sans intérêt. Il s'agit moins de remettre en cause la pertinence du concept-PME que d'évaluer de façon critique son degré de généralité. Cette orientation de recherche permet de concilier à la fois un fort degré d'autonomie de recherche par rapport aux travaux sur la grande entreprise (point faible du courant de la spécificité) et un fort degré de généralisation (réfutation) de ces résultats (point faible du courant de la diversité) sans toutefois faire de la spécificité de la PME une loi universelle ou un postulat (point faible du courant de la synthèse).

GRAPHIQUE 2

Degré de généralisation et autonomie de recherche des divers courants en PME

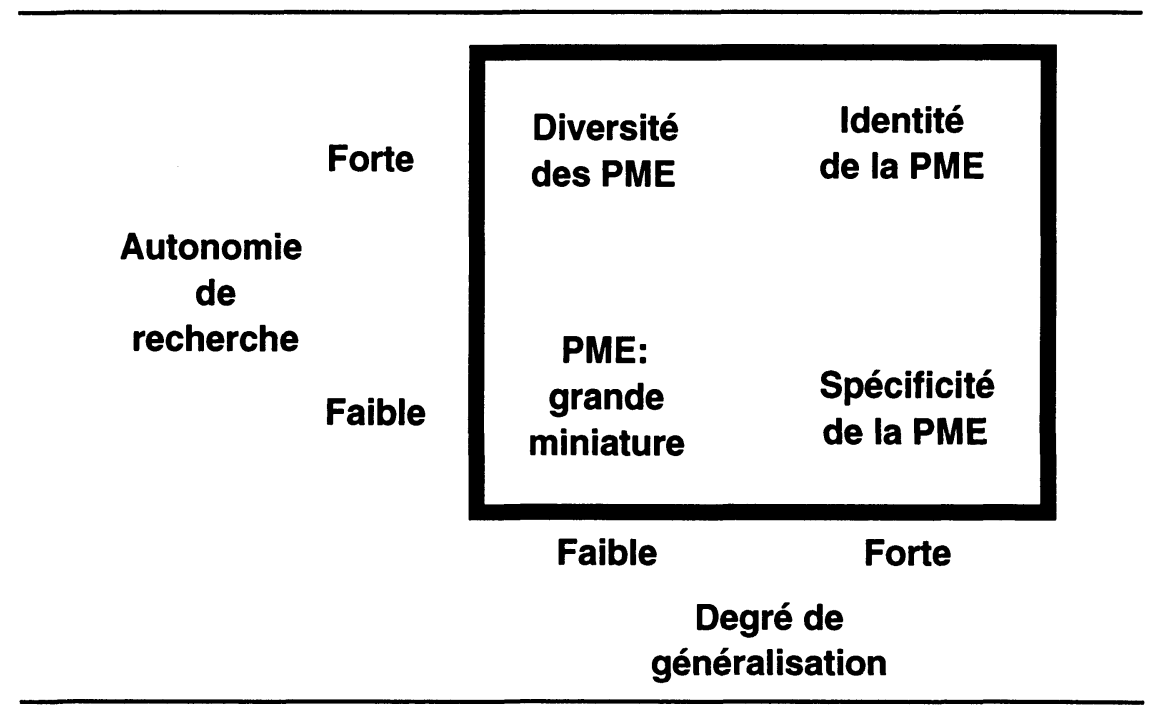




\section{Conclusion}

Considéré durant les années 1970 et 1980 comme un concept précis et universel, l'objet-PME devient, dans le courant des années 1990, une forme floue et contingente. Cette évolution semble conforme à la logique de la découverte scientifique telle qu'elle est décrite par Popper (1973): «Au début, nous devons suivre nos propres théories, car sans théorie nous ne pourrions commencer [...] Ensuite, nous devons adopter une attitude plus critique vis-à-vis de ce qui nous a permis d'avancer et essayer de les remplacer par des éléments plus adaptés, en fonction même de ce que nos propositions initiales nous ont permis d'apprendre.» Ce type de clivage (phase de proposition et d'examen critique) se retrouve dans les trois étapes qui ponctuent la recherche en PME. À chaque phase de généralisation théorique succède une phase plus critique et plus nuancée à l'égard des travaux initiaux.

TABLEAu 1

Évolution historique de la recherche en PME

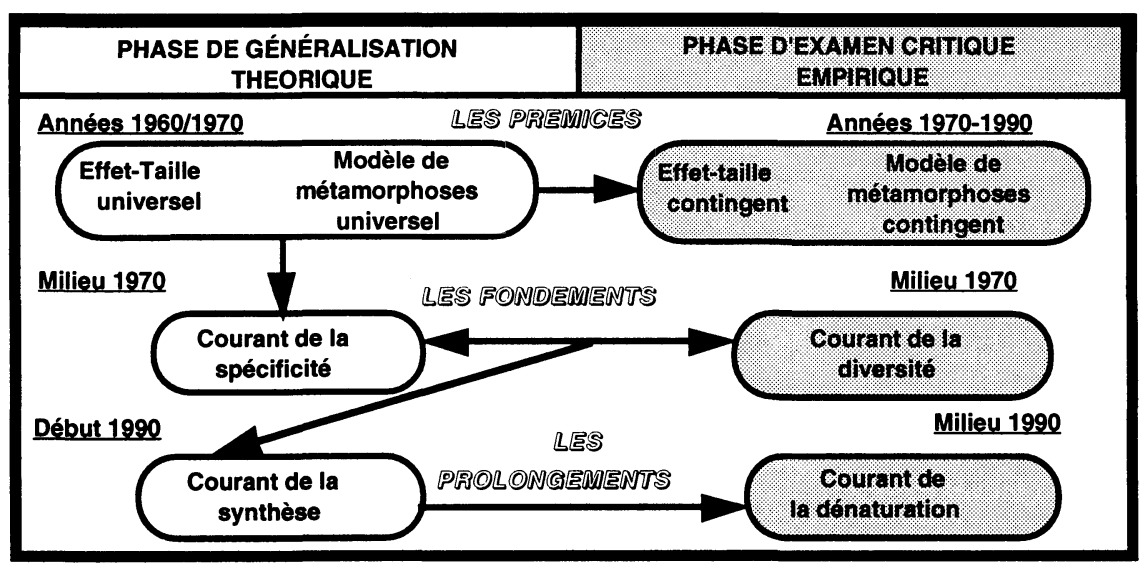

Ce tableau montre la diversité et l'enchaînement des courants de recherche en PME depuis ces vingt dernières années. Chaque courant contribue à fournir aux courants suivants des éléments de base pour faire progresser l'état des connaissances de la PME. Il permet de prendre conscience de tous les courants, travaux antérieurs qui constituent très souvent des acquis ou les bases sur lesquels les courants successifs se sont fondés. L'analyse historique explicite les hypothèses qui, avec le temps et l'oubli, deviennent rapidement des postulats implicites ou ignorés des jeunes chercheurs qui «prennent le train en marche». Ce classement fournit alors une grille d'analyse utile pour positionner des travaux récents dans le cadre plus général de la recherche en PME. Ce tableau 
montre également que la recherche en PME est constamment traversée par le clivage entre universalisme et contingence. Cette alternance permanente impliquerait alors que le courant de la dénaturation soit destiné à se développer de plus en plus. D'une part, le schéma montre clairement que ce courant hérite des travaux des courants de la spécificité et de la diversité. D'autre part, il permet de tenir compte des récents amendements concernant les travaux portant sur les modèles de croissance. Enfin, en identifiant les contextes incompatibles à la forme-PME, ce courant contribue à mieux cerner le cadre de validité du paradigme de la spécificité et donc l'identité de la PME.

L'autre intérêt de cette orientation de recherche est de permettre l'analyse d'éléments récents et nouveaux qui n'existaient pas lors de la genèse du modèle. Dans un article de synthèse sur les travaux empiriques canadiens concernant les PME depuis 1980, d'Amboise (1993), déplorant le caractère fragmenté du corpus théorique en PME, considère que la recherche en PME ne progressera qu'à la condition de prendre en compte les nouvelles tendances de l'économie et des pratiques des entreprises, et en comparant les effets de différents contextes sur un corpus théorique unitaire. Dans son ouvrage Les sciences de l'imprécis, à propos de l'attitude critique inhérente à l'esprit scientifique, Moles (1990) note :

Le travail d'une théorie scientifique est long, et dans l'intervalle, de nouveaux éléments se sont proposés pour s'ajouter à son corpus. Dans quelle mesure pourra-t-il les y faire entrer sans changer les règles du jeu telles qu'il les a fixées (validité externe) ? En général, il sera conduit à modifier ces règles mais il cherchera encore quel est le nombre minimal de changements ou de règles additionnelles qu'il devrait ajouter à son répertoire pour faire face à une réalité toujours submergeante.

Nous posons donc la question suivante : dans quelle mesure la conception traditionnelle de la PME élaborée à la fin des années 1970 est apte à intégrer certains phénomènes nouveaux ? Le courant de la dénaturation constitue un prolongement de la recherche en PME dans une perspective contingente puisqu'il s'inscrit pleinement dans la phase d'examen critique telle qu'elle est décrite par Moles. Il nous semble que cette démarche présente l'avantage de contribuer conjointement à l'approfondissement et à l'accumulation des connaissances sur la PME, car il s'agit d'examiner de façon critique le paradigme de la spécificité qui sert néanmoins de base à l'analyse.

En définitive, si la recherche en PME a réussi à affirmer son identité épistémologique durant ces vingt dernières années, force est de constater qu'elle est fondée sur un projet cognitif nécessairement flou et instable et dont les frontières sont nécessairement contingentes. C'est la raison pour laquelle nous plaidons en faveur d'une approche contingente de l'objet-PME. 


\section{Bibliographie}

BARREYRE, P.Y. (1967), L'horizon économique des petites et moyennes entreprise, Thèse pour le doctorat de sciences économiques, Université de Grenoble, 480 p.

BAUER, M. (1993), Les patrons de PME, entre le pouvoir, l'entreprise et la famille, Paris, Inter Éditions.

BAUer, M. (1995), «PME: un patronat aux trois visages », Sciences Humaines, $\mathrm{n}^{\circ} 48$, p. 34-37.

BAYAD, M et D. NEBENHAUS (1994), « Recherches sur la GRH en PME : proposition en vue d'un modèle théorique ", Communication au Ve Congrès de l'AGRH, Montpellier, p. 235-242.

Bayad, M., H. Mahé de Boislandelle, D. Nebenhaus et P. Sarnin (1995), « Paradoxe et spécificités des problématiques de gestion des ressources humaines en petites et moyennes entreprises », Gestion 2000, $\mathrm{n}^{0} 1$.

Belletante, B et J. Desroches (1994), «L'impact de la cotation en bourse sur le comportement financier des moyennes entreprises ", Annales du Management, XII' Journées nationales des IAE, Montpellier, p. 309-329.

BERNARD, J et J.L. RAVIX (1988), «Diversité et spécificité des unités productives: les clivages par taille et les typologies d'entreprises », dans R. Arena et al. (éd.), Traité d'Économie Industrielle, Paris, Economica, p. 192-208.

BERNARD, J.P. (1994), «Synthèse des thèses récentes en management stratégique et politique générale ", Annales du Management, XII ${ }^{\mathrm{e}}$ Journées des IAE, Montpellier, p. 29-38.

BLAU, P.M. (1970), «A formal theory of differenciation in organizations », American Sociological Review, vol. 35, $\mathrm{n}^{0} 2$, p. 201-218.

BlaU, P.M. et R. SCHOENHERR (1971), The Structure of Organizations, New York, Basic Books.

Bournois, F et C. PELLEgRIN (1994), « De la PMI à la grande entreprise internationalisée : un cas de conservation des principales caractéristiques organisationnelles de la PMI originelle», Actes présentés à la $39^{\circ}$ Conférence mondiale de l'ICSB, «Les PME/PMI et leur contribution au développement régional et international», 27-29 juin, Strasbourg, p. 49-53.

BROOKSBANK, R. (1991), "Defining the small business: a new classification of company size », Entrepreneurship and Regional Development, $\mathrm{n}^{\circ} 3$, p. 17-31.

BRYTTING, T. (1991), «Organizing in the small growing firm: a grounded theory approach », The Economic Research Institute/EFI, Stockholm, 238 p.

CANDAU, P. (1981), «Pour une taxonomie de l'hypofirme », Revue d'Économie Industrielle, $\mathrm{n}^{\circ} 16$, p. 16-33. 
Chicha, P., P.A Julien et M. MARChesnay (1990), «Processus et procédures stratégiques en PME», International Gewerbearchiev, vol. 38, $\mathrm{n}^{\circ} 3$.

CHILD, J. (1972), «Organization structure and strategies of control : a replication of the Aston study », Administrative Science Quarterly, vol. 17, p. 163-177.

ChILD, J et R. MANSFIELD (1972), «Technology, size and organization structure », Sociology, vol. 6, p. 369-393.

Churchill, N et V. Lewis (1983), «Les cinq stades de l'évolution d'une PME », Harvard L'Expansion, p. 51-63.

COHEN, E. (1989), «Épistémologie de la gestion », Encyclopédie de Gestion, Paris, Economica, p. 1055-1074.

Curvalle, B. (1994), «L'EDI et le JAT condamnent-elles les PME ?», Communication aux $\mathrm{II}^{\mathrm{e}}$ Journées IUT de la Recherche en sciences sociales et humaines, Toulouse.

D'Amboise, G. et G. Plante (1987), « La recherche sur la PME : quelques voies pour des relations efficaces entre chercheurs et dirigeants ", Revue de Gestion des petites et moyennes organisations, vol. $3, \mathrm{n}^{0} 1, \mathrm{p} .44-50$.

D'AMBoise, G. et M. MALDOWNEY (1988), « Management theory for small business : attempts and requirements ", Academy of Management Review, vol. 13, $\mathrm{n}^{\circ} 2$, p. 226-240.

D' AмвоISE, G. (1993), « Empirical research on SME's : the past ten years in Canada », Journal of Small Business \& Entrepreneurship, vol. 10, $\mathrm{n}^{\circ}$ 2, p. 2-12.

DANDRIDGE, T.C. (1979), « Children are not "little grown-ups" : small business needs its own organizational theory », Journal of Small Business Management, vol. 17, $\mathrm{n}^{\circ} 2$, p. 53-57.

DesreumauX, A. (1992), Structures d'entreprise, Paris, Vuibert Gestion, 334 p.

Dubost, N. (1995), «Les PME françaises évoluent-elles vers des bureaucraties?», Communication au Congrès de l'AIMS, Paris-Dauphine, p. 596-611.

FABI, B., D. GARAND et N. PETTERSEN (1993), «La gestion des ressources humaines en PME : proposition d'un modèle de contingence », Communication au Congrès international francophone de la PME, Carthage, 28-30 octobre, p. 216-229.

Gervais, M. (1978), «Pour une théorie de l'organisation-PME », Revue Française de Gestion, $\mathrm{n}^{\circ} 15$, p. 37-48.

GODENER, A. (1994), « Entreprises traditionnelles et entreprises de haute technologie, des chemins de croissance différents ? », Annales du Management, XII Journées nationales des IAE, tome 2, Montpellier, p. 175-192.

GODENER, A. (1995), «Les changements de dimensions de l'entreprise en croissance », Communication au Congrès de l'AIMS, Paris, p. 612-631. 
GREINER, L.E. (1972), «Evolution and revolution as organizations grow », Harvard Business Review, juillet-août, p. 37-46.

Guilhon, A., B. Guilhon et D. Peguin (1993), "L'identité de la PME à travers l'activité d'exportation », Communication au Congrès international francophone de la PME, Carthage, 28-30 octobre 1993, p. 301-312.

Guilhon, A., B. GuILHON et D. PEGUIN (1995), « PME, exportation et contrôlabilité », Communication au Congrès international francophone de la PME, Paris, 25-27 octobre, p. 385-401.

Guilhon, B. et M. MARCheSNAY (1994), «Présentation du nouveau développement en économie industrielle », Revue d'Économie Industrielle, numéro spécial, $\mathrm{n}^{0} 67$.

Hall, H. (1972), Organizations: Structure and Process, Englewood Cliffs, N.J., Prentice-Hall, 291 p.

HALl, H., J.E. HAAS et N.J. JoHNSON (1967), «Organizational size, complexity, and formalization », American Sociological Review, vol. 32, nº 6, p. 902-912.

Hertz, L. (1982), In Search of a Small Business Definition; An Exploration of the Small-business Definitions of U.S., the U.K., Israel and the People's Republic of China, Washington, University Press of America, 454 p.

Julien, P.A. et M. MARCheSnay (1988), La petite entreprise, Paris, Vuibert, 288 p.

JULIEN, P.A. et M. MARCHESNAY (1992), « Des procédures aux processus stratégiques dans les PME », p. 97-129 dans Perspectives en Management stratégique, tome 1: $1992 / 1993$, A. Noël (éd.), Paris, Economica-Gestion, 431 p.

JULIEN, P.A. (1993), «Small businesses as a research subject : some reflections on knowledge of small businesses and its effects on economic theory », Small Business Economics, vol. 5, p. 157-166.

JULIEN, P.A. (éd.) [1994], Les PME : bilan et perspectives, Paris, Economica, 352 p.

KAZANJIAN, R. et R. DRAZIN (1990), «A stage contingent model of design and growth for technology based new ventures », Journal of Business Venturing, $n^{0} 5$, p. $137-150$.

KIMBERLY, J.R. (1976), «Organizational size and the structuralist perspective : a review, critique, and proposal», Administrative Science Quarterly, vol. 21, p. 571-597.

LECLERC, Y. (1990), «De la sous-traitance au partenariat: le Japon, "modèle" de référence?», Communication au Colloque TETRA sur le thème «La PME: objet de recherche pertinent?», 30-31 mai, Lyon.

LÉO, P.Y. (1987), «Les milieux régionaux de PMI : une approche statistique et régionalisée des choix stratégiques des PMI à partir de l'EAE », Revue d'Économie Régionale et Urbaine, $\mathrm{n}^{\circ} 3$, p. 423-437.

MAhÉ De Boislandelle, H. (1994), «Esquisse d'une théorisation de la GRH de la PME », Communication au Congrès de l'AGRH, Montpellier, p. 259-269. 
MARCH, J.G. (1994), «Tout essai de description des tendances de la gestion ne décrit de façon adéquate aucune situation de gestion particulière », Revue Française de Gestion, no 100 , p. 22-24.

MARCHESNAY, M. (1982a), "Pour un modèle d'hypofirme », Entreprise et organisation, Mélanges en l'honneur du professeur Aubert-Krier, Paris, Economica, p. 71-91.

MARCHESNAY, M. (1982b), «Is small so beautiful ? », Revue d'Économie Industrielle, $\mathrm{n}^{\circ} 19$, p. $110-114$.

MARCHESNAY, M. (1991), « De l'hypofirme à l'hypogroupe : naissance, connaissance, reconnaissance », Toulouse, Les Cahiers du Lerass, ${ }^{\circ}$ 23, p. 33-50.

MARCHESNAY, M. (1993), «PME, stratégie et recherche », Revue Française de Gestion, $\mathrm{n}^{\circ} 95$, p. 70-76.

MARTINET, A.C. (1986), «Pour une théorie des formes stratégiques : réflexions épistémologiques naïves », Strategia, Cahier de recherche de l'IAE de Lyon, 14 p.

MinTZBERG, H. (1982), Structure et dynamique des organisations, Paris, Éditions d'Organisation, $434 \mathrm{p}$.

Moles, A.A. (1990), Les sciences de l'imprécis, Paris, Éd. Seuil.

PEnRose, E.T. (1959), The Theory of the Growth of the Firm, Oxford, Basic Blackwell.

Perrat, J. (1990), « Développement des PME: les limites de l'approche statistique », Communication au Colloque TETRA sur le thème «La PME : objet de recherche pertinent?», 30-31 mai, Lyon.

POPPER, K. (1973), La logique de la découverte scientifique, Édition originale allemande 1936, traduction française, Paris, Payot.

Pugh, D.S., D.J. Hickson, C.R. Hinings et C. TuRnER (1968), «Dimensions of organization structure », Administrative Science Quarterly, vol. 13, n 1, p. 65-105.

PUGH, D.S., D.J. HICKSON et C.R. HININGS (1969), « An empirical taxonomy of structure of work organizations », Administrative Science Quarterly, vol. 14, p. 115-126.

SARNIN, P. (1990), "Changements stratégiques et pratiques de formation dans 16 PMI de Rhône-Alpes », Communication au Colloque TETRA «La PME : objet de recherche pertinent?», 30-31 mai 1990, Lyon.

STEPHANY, E. (1993), Contribution à l'étude de la liaison structure du capital, relation d'agence et performance financière: le cas des PME familiales d'Aquitaine financées par capital risque développement, Thèse de doctorat, Université de Bordeaux I, 378 p.

WeLSH, J.A et J.F. White (1981), «A small business is not a little big business », Harvard Business Review, vol. 59, $\mathrm{n}^{0} 4$, p. 18-32. 